\title{
Effect of ischemia preconditioning on renal ischemia/ reperfusion injury in rats
}

\author{
Lian-hui Fan, Long He, Zhi-giang Cao, Jun Xiang, Long Liu \\ Department of Urology, The General Hospital of Shenyang Military Region, Shenyang, China
}

\section{ABSTRACT}

Objective: To study the effect of ischemia preconditioning (IP) on renal ischemia/reperfusion (I/R)-associated functional injury and expression of renal adhesion molecules in rats.

Materials and Methods: The ischemia preconditioning plan adopted in this experiment involved renal warm ischemia for $6 \mathrm{~min}$. and blood flow for $4 \mathrm{~min}$., repeated four times. The Wistar rat kidneys used for warm ischemia preconditioning were subjected to 60 min of renal warm ischemia followed by reperfusion. The rat kidneys with ischemia/ reperfusion were compared with the ischemia preconditioning group to observe rat renal function and changes in the expression of renal adhesion molecules ICAM-1, P-Selectin, and E-Selectin.

Results: The expression of rat renal adhesion molecules (ICAM-1, P-Selectin, and E-Selectin) with ischemia preconditioning was significantly lower than that of the ischemia/ reperfusion group. Serum creatinine was significantly lower than that in the ischemia/ reperfusion group after 48 hours.

Conclusions: Ischemia preconditioning has a protective effect on renal function. Reduced expression of renal adhesion molecules is likely a mechanism involved in the observed protection.

\section{ARTICLE INFO}

\author{
Key words: \\ Ischemic Preconditioning; \\ Intercellular Adhesion \\ Molecule-1; P-Selectin; \\ E-Selectin; Ischemia; \\ Reperfusion Injury
}

Int Braz J Urol. 2012; 38: 842-54

Submitted for publication:

May 10, 2012

Accepted after revision:

October 10, 2012

\section{INTRODUCTION}

Acute kidney injury (AKI) with high mortality and morbidity occurs frequently in critical patients. Ischemia is currently the leading cause of AKI in hospitalized patients. Kidney ischemic/ reperfusion (I/R) injury is the major cause of renal injury in ischemic AKI. Avoiding IRI is very difficult in organ transplantation. The occurrence mechanism of kidney $\mathrm{I} / \mathrm{R}$ injury is complex and data show that inflammatory mediators, adhesion molecules, and a variety of cytokines are involved in kidney I/R injury, but the exact pathogenesis has not been elucidated. If the condition in AKI cannot be improved in time, $\mathrm{I} / \mathrm{R}$ injury may be persistent for several weeks or even years and evolves always to irreversible chronic kidney disease (CKD).

Ischemia preconditioning (IP) refers to an effective protective measure produced by tissues and organs after a brief ischemia/reperfusion, which can produce tolerance to ischemia-reperfusion injury (1-3). Research shows that preconditioning stimulation can protect the target organs or distant parts of organs and tissues $(4,5)$. This phenomenon can be observed in many organs, such as skeletal muscle (6) and the heart (4). In the early stage, kidney tolerance can be induced by applying intermittent blood flow interruptions in the early phase of reperfusion (7). The kidney is a vital organ in which preconditioning can probably produce a protecti- 
ve effect. Given the need for high energy and a complex vascular network, kidneys are extremely sensitive to ischemia/reperfusion $(8,9)$. Kidney $I / R$ injury is also an important cause of the delayed recovery of function after renal transplantation (10-12). Animal experiments have confirmed that local and distant preconditioning are effective in protecting the kidneys $(13,14)$. Meta-analysis indicated that IP can reduce serum creatinine, blood urea nitrogen and histological renal damage after kidney I/R injury as compared to controls, suggesting that IPC effectively reduces renal damage after kidney I/R injury. Increased secretion of adhesion molecules after renal injury caused adhesion of inflammatory cells to endothelial cells, resulting in release of multiple cytokines. These cytokines can also enhance expression of adhesion molecules which not only may result in aggravation of renal injury but also make the rejection of transplanted organ occurs or aggravates. Nowadays, most studies are limited to the macroscopic analysis of the protective effect of renal function. The mechanism behind the renal ischemia preconditioning phenomenon is yet to be elucidated.

The inflammatory cascade caused by ischemia/reperfusion is an important factor leading to renal I/R injury. Research has shown that leukocyte activation, invasion, adhesion, and impaction evidently occur in the tissue of an AKI model. As a result, perfusion is difficult to recover, and a variety of hazardous substances (such as free radicals, lysosomal enzymes, and various cytokines) is released, causing histiocyte damage $(15,16)$. The adhesion of leukocytes to the endothelium is mainly mediated by intercellular adhesion molecule- 1 . Previous studies have confirmed that the expression of adhesion molecules increases after kidney I/R injury (17-20). This paper established an ischemia/reperfusion disease model in rat and studied the expression level of adhesion molecules in IP. The relationship between adhesion molecule expression and kidney I/R injury after IP was also investigated.

\section{MATERIALS AND METHODS}

\section{Animals}

One hundred and fifty 2- to 3-month-old Wistar rats (200 g to 250 g; male or female; Third
Military Medical University Animal Institute) were used in this experiment. Goat anti-rat ICAM-1 antibody and goat anti-rat P-Selectin antibody (Beijing Zhongshan Biotech Co., Ltd.,. China); Goat anti-rat E-Selectin antibody (Ancell, USA); ASBC immunohistochemistry (IHC) kit (Boster Bio-Engineering Co., Ltd. Wuhan, China); Biotinylated Goat Anti Rabbit IgG (Boster Bio-Engineering Co., Ltd. Wuhan, China); Rat Creatinine (Cr) ELISA Kit (Shanghai Xitang Biological Technology Co., Ltd., China) were also used. This study was carried out in strict accordance with the recommendations in the Guide for the Care and Use of Laboratory Animals of the National Institutes of Health. The animal use protocol has been reviewed and approved by the Institutional Animal Care and Use Committee (IACUC) of The General Hospital of Shenyang Military Region.

\section{Experimental Methods}

The Wistar rats were randomly divided into three groups: sham-operated, ischemia/reperfusion (I/R), and IP groups. Each group was further sorted by time point at 1,2,12, 24, and 48 hours (each subgroup with 10 rats). Sodium pentobarbital (1\%) was given to the rats via intraperitoneal anesthetic injection (30 40 mg/kg). The abdominal cavity was opened under aseptic conditions. The right kidney was excised, and the left renal pedicle was freed. In the sham-operated group, the incisions were closed immediately after $60 \mathrm{~min}$. In the ischemia/reperfusion group, the left renal artery and veins were occluded for $60 \mathrm{~min}$. using microaneurysm clamps, and then the incisions were released and closed. In the IP group, the renal artery and veins were occluded for $6 \mathrm{~min}$ using microaneurysm clamps and the blood was allowed to flow again for $4 \mathrm{~min}$. The whole procedure was repeated four times. After these procedures were conducted the renal artery and veins were occluded for $60 \mathrm{~min}$. After the warm ischemia, the microaneurysm clamps were released, and the incisions were closed.

Rats in each group were euthanized by cervical vertebra luxation while still under anesthesia and samples were collected from their blood, tissues at 1, 2, 12, 24, and 48 hours after the incisions were closed. The left renal biopsy specimens 
were taken and fixed in mercuric chloride (4.5 $\mathrm{mL})+$ formaldehyde $(0.5 \mathrm{~mL})$ solution for $5 \mathrm{~min}$., and then stored at $4{ }^{\circ} \mathrm{C}$ in picric acid $(5 \mathrm{~mL})$ until pathological examination. Venous blood was collected to determine the level of serum creatinine in the vena cava at the time of sacrifice.

\section{Immunohistochemical staining}

All the renal tissue specimens were dehydrated by automatic tissue dehydration machine, paraffin embedded, cut into $5 \mu \mathrm{m}$ sections and mounted onto glass slides. Two percent (v/v) APES in acetone was used as adhesive for fixed tissue. At least six pieces of sections were cut in each specimen: one of the slides was stained with $\mathrm{HE}$ and the rest of specimens were placed at 70 ${ }^{\circ} \mathrm{C}$ overnight. Immunohistochemical staining was performed according to the product manual. Each kidney specimen was stained with ICAM-1, P-Selectin, and E-Selectin antibodies for 10 sections, with three sections extracted randomly thereafter. The concentrations of the primary and secondary antibodies were 1:100 dilution. PBS was used as negative control. The specimens were stained with SABC method. Briefly, the specific steps are as follows (ICAM-1 as an example). (1) slice conventional dewaxing to water progressively: Xylene (I), 5 min.; Xylene (II), 5 min.; 100\% ethanol, 95\% ethanol, 90\% ethanol, 85\% ethanol, 75\% ethanol, 5 min. each; rinse in distilled water, 5 min. (2). 1\% hydrogen peroxide was used to treat slices at RT for $15 \mathrm{~min}$. to eliminate endogenous peroxidase activity (3). Wash with distilled water and soak in PBS 3 times for 5 min. each (4). Antigen retrieval in microwave for $11 \mathrm{~min}$. Gradually cooled to RT and soak in PBS for 5 min. (5). Non-immune serum of normal animal block at RT for 15 min. to reduce non-specific staining (6). Dumping of serum and dropping goat anti-rat ICAM-1 antibody (1:100 dilution). Incubate at $37^{\circ} \mathrm{C}$ for 1 hour and overnight at $4{ }^{\circ} \mathrm{C}(7)$. Wash with $0.01 \mathrm{M}$ PBS 3 times for 5 min. each (8). Dropping Biotinylated Goat Anti Rabbit IgG and incubation at $37^{\circ} \mathrm{C}$ for 1 hour (9). Wash with 0.01M PBS 3 times for 5 min. each (10). Add avidin and incubate at $37{ }^{\circ} \mathrm{C}$ for 1 hour (11). Wash with 0.01M PBS 3 times for 5 min. each (12). DAB color developed for $5 \mathrm{~min}$. Terminate the chromogenic reation until the yellow particles were observed in the cells under microscope (13). The nuclei were stained with hematoxylin (14). Wash with water for 5 min. Dehydrate with graded alcohol series (70\% ethanol, 80\% ethanol, 90\% ethanol, 95\% ethanol, 100\% ethanol, 5 min each). Clarify with Xylene for 15 min. Mounting with neutral resin.

The extent of renal injury was evaluated by counting the number of neutrophils infiltration in renal tissue. Three sections were randomly selected for counting the number of neutrophils in five adjacent high-power fields. The average was defined as the number of positive cells (neutrophils) for each detection indicator (Figure-1).

\section{Statistical analysis}

SPSS was used to analyze all data. The results were expressed as mean \pm SD. Statistical significance of differences among groups (defined as $\mathrm{P}<0.05$ ) was evaluated using one-way analysis of variance (ANOVA) with Bonferoni correction and Student's t-test.

\section{Figure 1 - The results for the histological examination. A) Control; B) IR pathology; C) IP.}

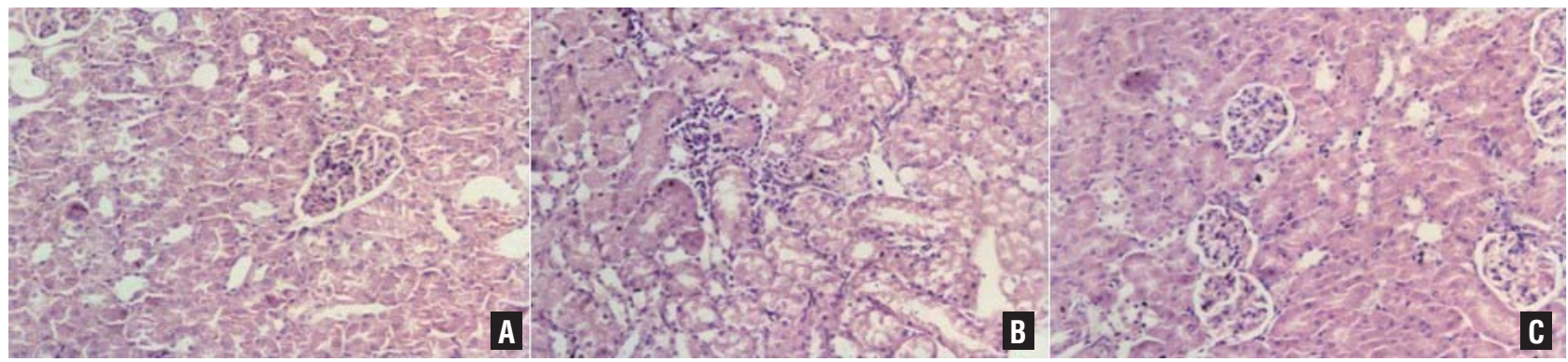




\section{RESULTS}

Changes in ICAM-1 expression after renal IP

A few positive cells were observed in the sham-operated group. Immunohistochemistry showed that renal ICAM-1 expression was enhanced after ischemia/reperfusion (Figure-2). The number of ICAM-1-positive cells increased at 2 hours after ischemia/reperfusion and peaked at 12 hours. A downward trend was observed at 24 hours. The expression of ICAM-1 was mainly observed in the glomerular endothelial cells, peritubular capillaries, and renal proximal tubule epithelial cells. After IP, the number of ICAM-1-positive cells decreased correspondingly. The expression level of ICAM-1 in the IP group was less than that in the I/R group at each time point ( $p<$ 0.05; Table-1 and Figure-3).
Changes in P-Selectin expression after renal IP

The number of positive cells in the sham-operated group was extremely few (Figure-4). One hour after ischemia/reperfusion, P-Selectin was widely expressed in the kidney, particularly in the glomerular mesangial, capillary loop, tubular, and renal interstitial sections. The expression was most significant in the renal tubular epithelial cells. It peaked 12 hours later and then declined. P-Selectin expression decreased significantly after IP ( $p<0.05$; Table-2 and Figure-5).

\section{Changes in E-Selectin expression after renal IP}

E-Selectin was rarely expressed in the sham-operated group (Figure-6). Two hours after ischemia/reperfusion, the cells expressing E-Selectin increased, specifically those in the peritubular capillaries. The expression peaked at

Figure 2 - Histology figures of ICAM-1 expression in the three groups. A) Control; B) IR; C) IP.

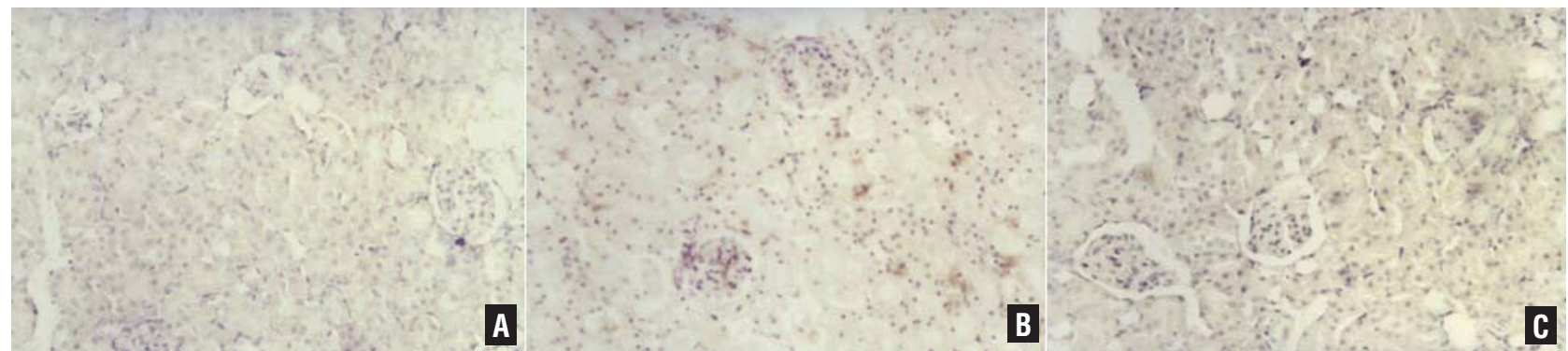

Table 1 - Effects of ischemic preconditioning on the expression of ICAM-1 in rat kidney (positive cell number/HP).

\begin{tabular}{|c|c|c|c|c|c|}
\hline & $1 \mathrm{~h}$ & $2 \mathrm{~h}$ & $12 \mathrm{~h}$ & $24 \mathrm{~h}$ & $48 \mathrm{~h}$ \\
\hline Sham-operated & $0.6 \pm 0.70$ & $1.0 \pm 0.67$ & $1.2 \pm 0.92$ & $1.1 \pm 0.74$ & $1.2 \pm 0.63$ \\
\hline $\mathrm{I} / \mathrm{R}$ & $2.1 \pm 0.99$ & $7.6 \pm 1.43 \boldsymbol{\Delta}$ & $12.9 \pm 2.33 \boldsymbol{\Delta}$ & $10.6 \pm 2.59 \boldsymbol{\Lambda}$ & $7.1 \pm 1.37 \boldsymbol{\Delta}$ \\
\hline IP & $1.1 \pm 0.74$ & $5.0 \pm 1.63 \Delta *$ & $10.4 \pm 1.43 \mathbf{\Delta} *$ & $8.3 \pm 1.49 \Delta *$ & $5.2 \pm 1.31 \boldsymbol{\Delta} *$ \\
\hline $\mathrm{F}$ & 4.3366 & 32.1898 & 68.4419 & 38.8833 & 34.0986 \\
\hline$P$ & 0.0383 & 0.0000 & 0.0000 & 0.0000 & 0.0000 \\
\hline
\end{tabular}

$\Delta \mathrm{P}<0.01$ vs. sham-operated; $* \mathrm{P}<0.05$ vs. I/R. 
Figure 3 - Effects of ischemic preconditioning on the expression of ICAM-1 in rat kindney.

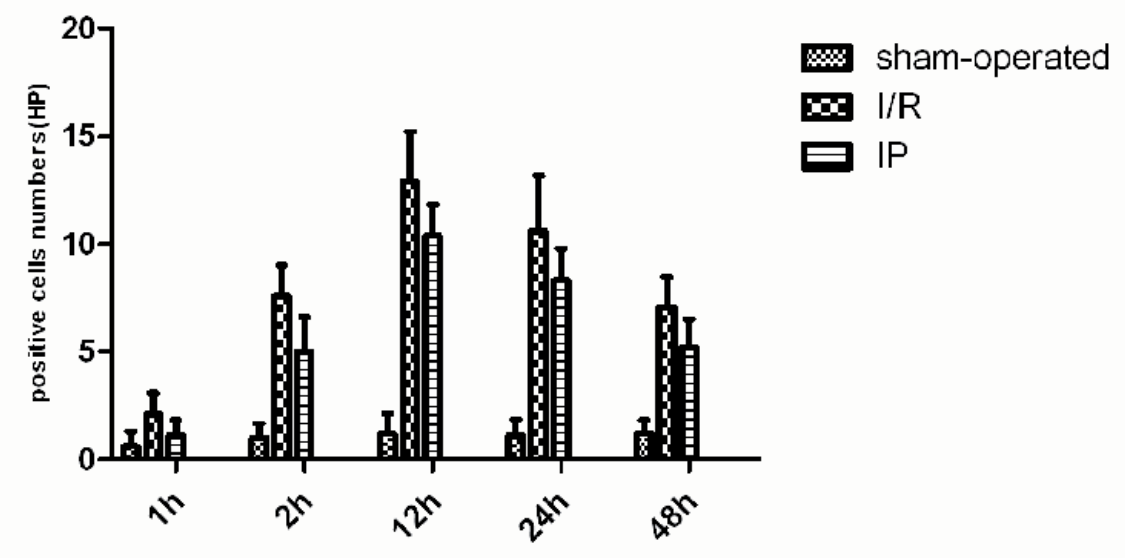

Figure 4 - Histology figures of P-Selectin expression in the three groups. A) Control; B) IR; C) IP.

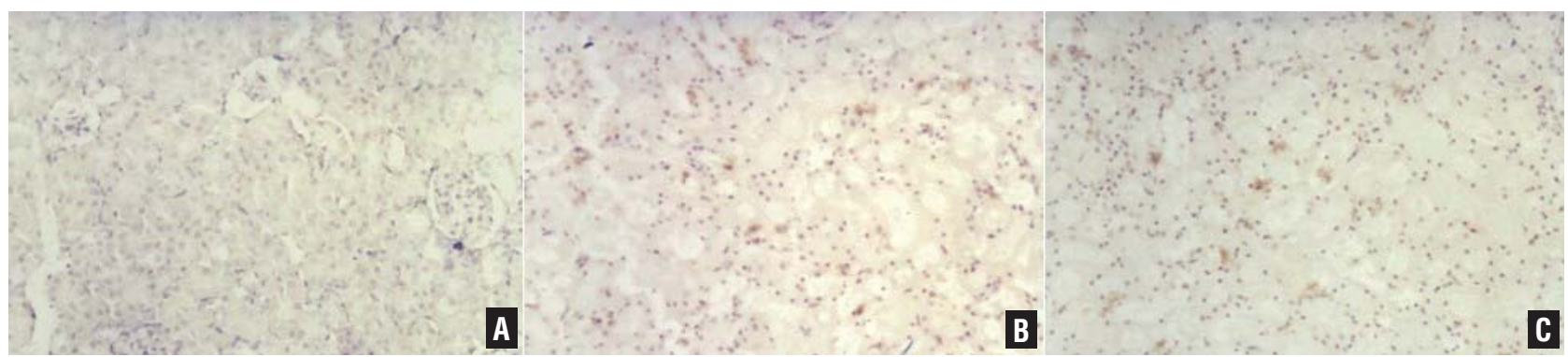

Table 2 - Effects of ischemic preconditioning on the expression of P-Selectin in rat kidney (positive cell number/HP).

\begin{tabular}{lccccc}
\hline & $1 \mathrm{~h}$ & $2 \mathrm{~h}$ & $12 \mathrm{~h}$ & $24 \mathrm{~h}$ & $48 \mathrm{~h}$ \\
\hline Sham-operated & $1.5 \pm 0.85$ & $1.5 \pm 0.85$ & $1.3 \pm 0.67$ & $1.2 \pm 0.63$ & $1.5 \pm 0.85$ \\
I/R & $6.0 \pm 1.15 \boldsymbol{\Lambda}$ & $9.9 \pm 1.52 \boldsymbol{\Delta}$ & $13.0 \pm 2.53 \boldsymbol{\Delta}$ & $4.7 \pm 1.16 \boldsymbol{\Delta}$ & $3.89 \pm 0.94$ \\
IP & $5.4 \pm 1.26 \boldsymbol{\Delta}$ & $6.4 \pm 1.65 \boldsymbol{\Delta} *$ & $10.8 \pm 1.81 \boldsymbol{\Delta} *$ & $4.4 \pm 1.65 \boldsymbol{\Delta}$ & $3.78 \pm 1.33$ \\
F & 24.6518 & 46.3999 & 57.2739 & 12.6428 & 8.0908 \\
$\mathrm{P}$ & 0.0001 & 0.0000 & 0.0000 & 0.0001 & 0.0060 \\
\hline
\end{tabular}

$\mathrm{P}<0.01$ vs. sham-operated; ${ }^{*} \mathrm{P}<0.01$ vs. I/R. 
Figure 5 - Effects of ischemic preconditioning on the expression of P-Selectin in rat kidney.

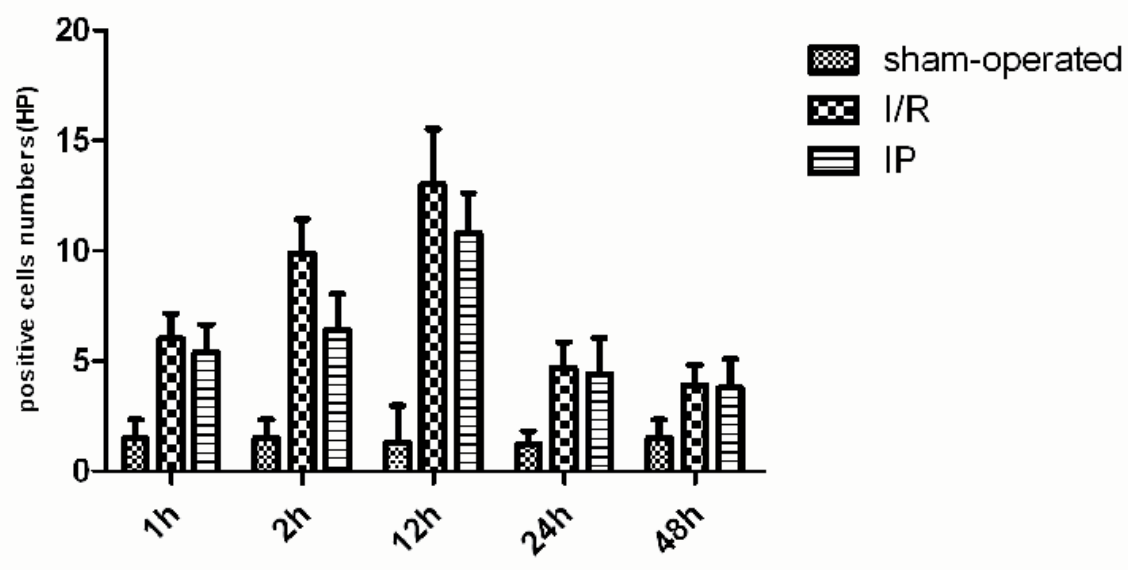

Figure 6 - Histology figures of E-Selectin expression in the three groups. A) Control; B) IR; C) IP.

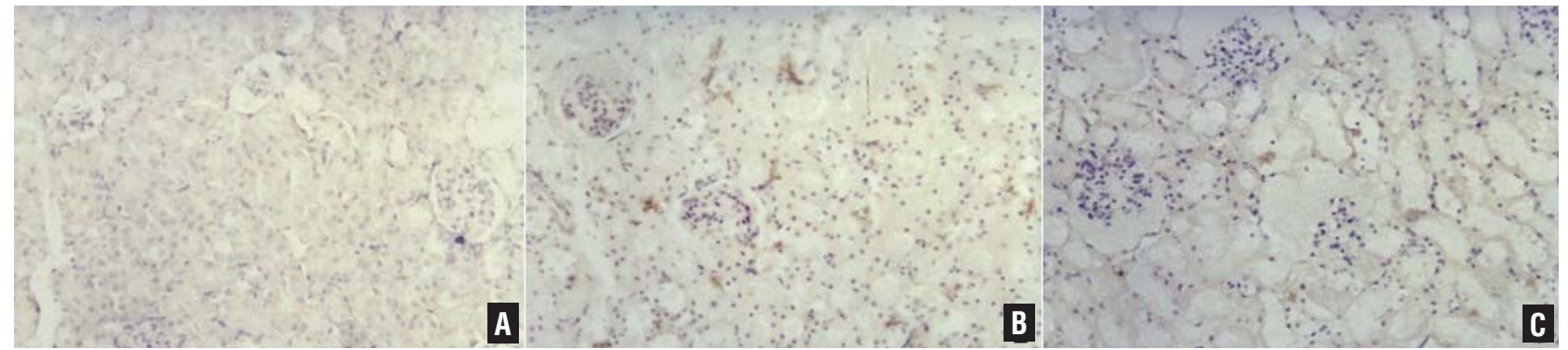

24 hours and then declined gradually. The number of positive cells in the IP group was significantly reduced ( $\mathrm{p}<0.05$; Table-3 and Figure-7).

Changes of leukocyte infiltration in renal tissues

One hour after ischemia/reperfusion, the number of leukocyte infiltration increased obviously. The number peaked at 24 hours and was significantly reduced at 48 hours. The infiltration occured mainly in vascular, interstitial and tubular areas of the kidney. Compared with those in IR group, the number of leukocyte infiltration was significantly reduced, especially at 24 hours ( $p<$ 0.05; Table-4 and Figure-8).

Renal ischemia/reperfusion and changes in renal function after IP

Serum creatinine levels increased after renal ischemia/reperfusion. In the first 24 hours, serum creatinine level was significantly higher than at 1, 2, 6, and 12 hours. ARF (acute renal failure) occurred when the creatinine level reached $140 \mu \mathrm{moL} / \mathrm{L}$ at the first 48 hours. By contrast, the creatinine level in the IP group also increased but at a slower rate. Compared with each corresponding time point of IP, no significant difference was observed at the first 1, 2, 12, and 24 hours. Compared with the IP group at the first 48 hours, the difference was significant ( $p<0.05$;Table-5 and Figure-9).

\section{DISCUSSION}

Preconditioning is a tolerance or adaptability to a secondary stimulus exhibited by organisms and organs after primary exposure to a stimulus. This phenomenon known as IP is common in biology. Organs can stand a longer time of 
Table 3 - Effects of ischemic preconditioning on the expression of E-Selectin in rat kidney (positive cell number/HP).

\begin{tabular}{lccccc}
\hline & $1 \mathrm{~h}$ & $2 \mathrm{~h}$ & $12 \mathrm{~h}$ & $24 \mathrm{~h}$ & $48 \mathrm{~h}$ \\
\hline Sham-operated & $0.6 \pm 0.70$ & $1.0 \pm 0.82$ & $1.0 \pm 0.67$ & $1.0 \pm 0.57$ & $1.11 \pm 0.63$ \\
$\mathrm{I} / \mathrm{R}$ & $2.4 \pm 1.07$ & $3.8 \pm 0.79 \boldsymbol{\Delta}$ & $6.2 \pm 1.14 \boldsymbol{\Delta}$ & $13.56 \pm 1.75 \boldsymbol{\Delta}$ & $3.1 \pm 1.1 \boldsymbol{\Delta}$ \\
$\mathrm{IP}$ & $1.7 \pm 0.67$ & $3.2 \pm 1.32 \boldsymbol{\Delta}$ & $4.4 \pm 0.97 \boldsymbol{\Delta} *$ & $8.44 \pm 1.49 \boldsymbol{\Delta} * *$ & $2.8 \pm 1.23 \boldsymbol{\Delta}$ \\
$\mathrm{F}$ & 5.9267 & 10.7276 & 38.8934 & 106.6971 & 5.5342 \\
$\mathrm{P}$ & 0.0162 & 0.0021 & 0.0000 & 0.0000 & 0.0198 \\
\hline
\end{tabular}

A $\mathrm{P}<0.01$ vs. control; $* \mathrm{P}<0.05, * * \mathrm{P}<0.01$ vs. I/R.

Figure 7 - Effects of ischemic preconditioning on the expression of E-Selectin in rat kidney.

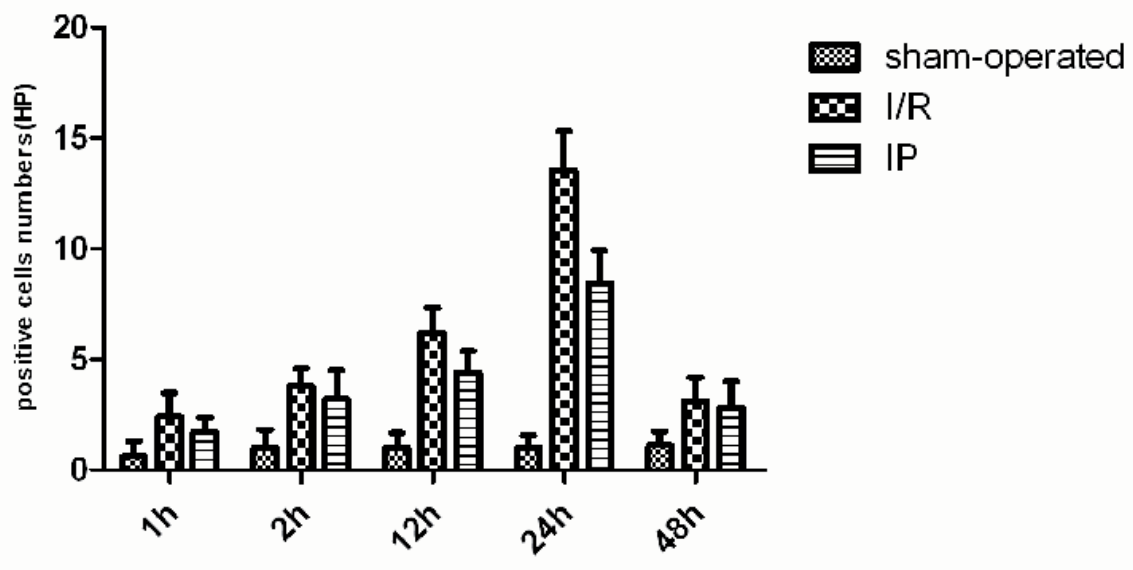

warm ischemia after brief ischemia/reperfusion. In 1986, Murry first observed the IP phenomenon in the heart (4). In later studies, many organs such as skeletal muscle have been demonstrated to exhibit IP (6).

In relation to renal preconditioning in a rat model, since the new concept of IP was proposed by Murry and protective effect of IP has been confirmed in cardiac ischemia-reperfusion injury, many scholars have established the heart IP model and explored its mechanism. If different preconditioning program was used in the renal IP model, the conclusions could not have been the same. Preconditioning involved the sequential clamping of the left renal artery for $4 \mathrm{~min}$. and its release for 11 min., a total of four times. The results of the above preconditioning program showed that no protection to kidney I/R injury was observed (21). However, Toosy et al. reported that an IP regimen applied only $5 \mathrm{~min}$. before exposure to a $40 \mathrm{~min}$. period of RI in the rat significantly protected it from the functional impairment associated with kidney I/R (22). Furthermore, the results of an IP regimen (three 2 min. periods of ischemia separated by $5 \mathrm{~min}$. of reflow prior to $45 \mathrm{~min}$. of bilateral renal ischemia followed by 
Table 4 - Changes of leucocyte Infiltrated into renal parenchyma after ischemic preconditioning.

\begin{tabular}{lccccc}
\hline & $1 \mathrm{~h}$ & $2 \mathrm{~h}$ & $12 \mathrm{~h}$ & $24 \mathrm{~h}$ & $48 \mathrm{~h}$ \\
\hline Sham-operated & $1.4 \pm 0.55$ & $1.8 \pm 0.84$ & $2.0 \pm 0.71$ & $1.6 \pm 0.89$ & $1.8 \pm 0.83$ \\
$\mathrm{I} / \mathrm{R}$ & $6.2 \pm 0.84 \boldsymbol{\Delta}$ & $11.4 \pm 2.30 \boldsymbol{\Delta}$ & $22.8 \pm 4.98 \boldsymbol{\Delta}$ & $32.6 \pm 4.40 \boldsymbol{\Delta}$ & $16.0 \pm 1.58 \boldsymbol{\Delta}$ \\
IP & $5.2 \pm 1.30 \boldsymbol{\Delta}$ & $7.8 \pm 1.30 \boldsymbol{\Delta} *$ & $13.6 \pm 2.07 \boldsymbol{\Delta} * *$ & $23.4 \pm 2.41 \boldsymbol{\Delta} * *$ & $8.8 \pm 1.82 \boldsymbol{\Delta} * *$ \\
$\mathrm{~F}$ & 35.6547 & 45.9040 & 55.0738 & 146.4627 & 116.3796 \\
$\mathrm{P}$ & 0.0000 & 0.0000 & 0.0000 & 0.0000 & 0.0000 \\
\hline
\end{tabular}

$\Delta \mathrm{P}<0.01$ vs. control; * $\mathrm{P}<0.05<* * \mathrm{P}<0.01$ vs. I/R

Figure 8 - Changes of leucocyte Infiltrated into renal parenchyma after ischemic preconditioning.

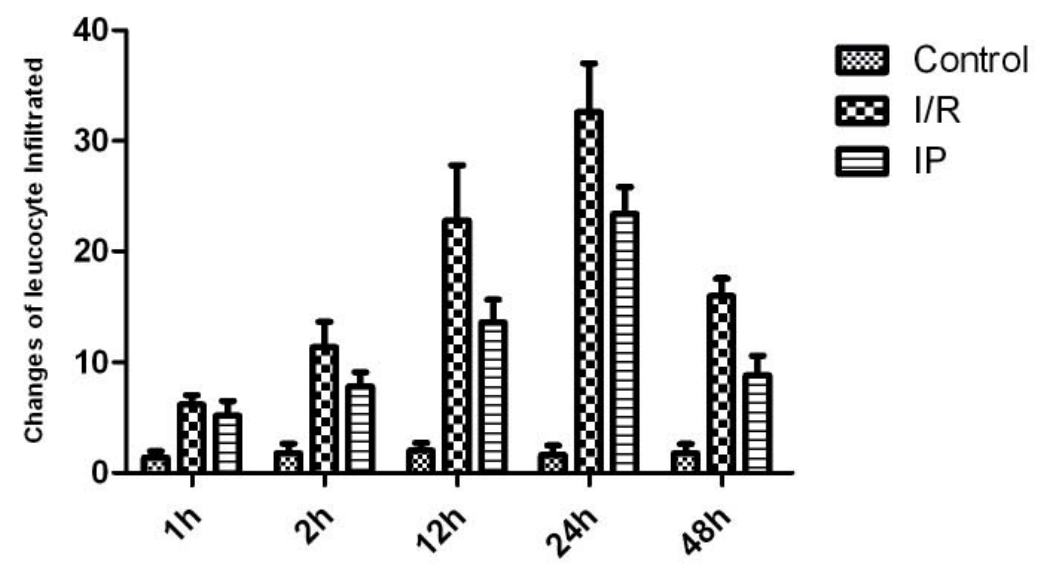

Table 5 - Changes of serum creatinine after renal ischemic preconditioning (umol/L).

\begin{tabular}{lccccc}
\hline & 1h & $2 \mathrm{~h}$ & $12 \mathrm{~h}$ & $24 \mathrm{~h}$ & $48 \mathrm{~h}$ \\
\hline Sham-operated & $57.0 \pm 3.16$ & $54 \pm 4.18$ & $62.4 \pm 2.65$ & $60.6 \pm 4.22$ & $61.8 \pm 5.67$ \\
I/R & $49.4 \pm 2.79$ & $55.4 \pm 5.59$ & $58.6 \pm 5.98$ & $79.8 \pm 7.6$ & $139.0 \pm 11.55 \boldsymbol{\Delta}$ \\
IP & $52.2 \pm 5.07$ & $54.6 \pm 6.10$ & $59.2 \pm 7.85$ & $75.2 \pm 7.99$ & $108.0 \pm 8.86 \mathbf{\Delta} *$ \\
F & 5.0972 & 0.0861 & 0.5996 & 10.8128 & 92.7601 \\
P & 0.0250 & 0.9180 & 0.5647 & 0.0021 & 0.0000 \\
\hline
\end{tabular}

$\Delta \mathrm{P}<0.01$ vs. sham-operated; $* \mathrm{P}<0.05$ vs. I/R. 
Figure 9 - Changes of serum creatinine after renal ischemic preconditioning.

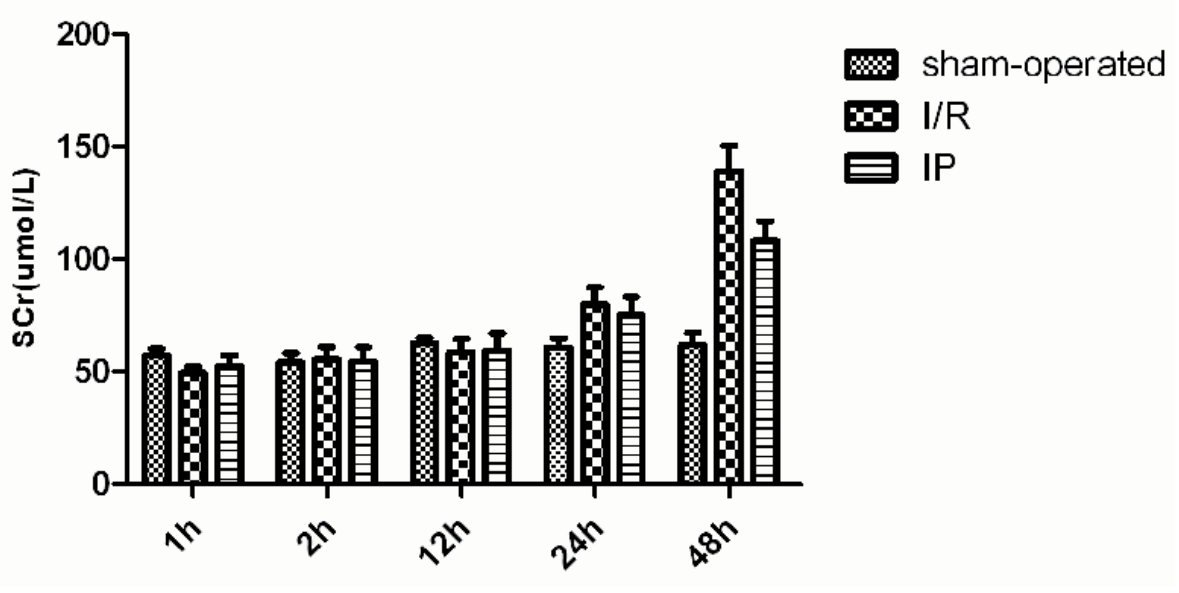

24 hours of reperfusion) applied by Cochrane et al. found that IP would ameliorate ischemic renal injury (23). The preliminary experiment of preconditioning method in our study found that the protective effect of prolongation of reperfusion time on renal I/R injury was not that obvious. The results of our preliminary experiment suggested that if the time and frequency of ischemia were fixed in a repeated cycle of short-time IR the protective effect on kidney would decline with the prolongation of the reperfusion interval. When a certain critical point is exceeded, such as fixed four $6 \mathrm{~min}$. periods of ischemia separated by more than $10 \mathrm{~min}$. of reperfusion, the role of IP protection would be lost. IP regimen with multiple cycles of short-period ischemia followed by short-period reperfusion had a significant protective effect on kidney I/R injury. At the same time, according to our previous experimental experience with I/R injury, an IP regimen applied 6 min. period of ischemia followed by 48 hours period of reperfusion was the most appropriate method to observe the renal function. Therefore, based on the previous study of the kidney IP model, the following modified IP regimen was used in the present study: the sequential clamping of the renal artery for $6 \mathrm{~min}$. and its release for 4 min., a total of four times; 60 min. of renal ischemia followed by 48 hours of reperfusion. The results of our study showed that this IP regimen may significantly reduce the incidence of renal I/R injury, suggesting that the experimental animal model was successfully established and may provide a reliable preadaptation program for further studies. According the observations of a preliminary study, changes of experimental data at 1, 2, 12, 24 and 48 hours after IP were obvious. Therefore, these time points were chosen as the observation point. Because one of the aims was to provide experimental data of IP protection for the kidney transplant, no prolonged observation time and the protective effect of later IP were further investigated in the present study.

Most studies have been limited to the macroscopic study of the protective effect of renal function in terms of the renal IP phenomenon. Wever conducted a meta-analysis of animal experimental studies on renal IP (24). Creatinine, urea nitrogen, and histological changes were used as indicators of renal IP. Meta-analysis showed that compared with the control group, serum creatinine and blood urea nitrogen levels decreased significantly and changes in the renal tissue was less important in the IP group. No difference was observed between local and distant preconditioning on the protective effect of renal ischemia/ reperfusion. IP significantly improved kidney I/R injury, especially in the advanced window period ( $\geq 24$ hours). Nevertheless, significant differences were found between studies in terms of the 
results of the best protection window. The effect of IP in each animal species varies. Indisputably, renal IP has protective effects to subsequent ischemic/reperfusion injury. However, the mechanism remains unclear. Mahfoudh-Boussaid (25) used Wistar rats to study IP and found that renal IP reduces cell lysis and lipid peroxidation and improves renal function. Compared with the ischemia/ reperfusion group, the IP group showed elevated levels of endothelial nitric oxide synthase, nitrite, and hypoxia inducible transcription factor- $1 \alpha$. Early preconditioning through reduction of oxidative and endoplasmic reticulum stress has been believed to protect against kidney I/R injury. Kim (26) believed that the effect of IP on the renal protective effect is related to the increase in isocitrate dehydrogenase (IDH1) activity. IDH1 enhanced NADPH levels, elevated the GSH-to-total glutathione ratio, and reduced oxidative stress. Chen (3) believed that NF-kappaB is the key medium to reperfusion injury. The activation of NF-kappaB depended on the phosphorylation of its inhibitor. The inhibitor was IkappaB, which had a specific NF-kappaB kinase subunit. Animal experiments confirmed that renal IP reduces renal acute injury by inhibiting IKKbeta activity. The IP protocol adopted in this experiment involved four repetitions of warm ischemia for $6 \mathrm{~min}$. and blood flow for $4 \mathrm{~min}$. Under the condition of 1 hour warm ischemia and 48 hours perfusion, IP significantly reduced the expressions of renal adhesion molecules ICAM-1, P-Selectin, and E-Selectin. At the same time, granulocyte infiltration in renal tissue after IP was significantly lower than that in the I/R group. Adhesion molecules are the major molecules which mediate adhesion of polymorphonuclear leukocytes to endothelial cells. Because of reduced expression of adhesion molecules after IR, adhesion of granulocytes to endothelial cells decreased leading to reduction of granulocyte infiltration in renal tissue. The serum creatinine level of the rats was significantly lower than that of the I/R group. This result proves the explicit protective effect of IP. The mechanism of kidney protection was closely related to the reduction of adhesion molecule expression caused by IP.

We believe that the reduction of adhesion molecule expression is one of the main mechanis- ms of IP in preventing ischemic/reperfusion injury. ICAM-1 distributes in a variety of cells. The degrees of ICAM-1 expression in different cells are not the same: the strongest is in vascular endothelium followed by peripheral blood leukocytes, interstitial macrophages and fibroblasts. Expression of ICAM-1 which is low in normal cell is enhanced by cytokines such as interleukin-1 (IL-1), tumor necrosis factor (TNF). Expression of ICAM-1 increased by the role of cytokines in the injured endothelial cells, resulting in promoting the adhesion of leukocytes to vascular endothelium and migration from intravascular to the extravascular matrix. E-selectin was expressed on the surface of the stimulated endothelial cells. There was no E-selectin in the endothelial cells. E-selectin was generated by transcription 4-6 hours after stimulation. Its function is to mediate the adhesion of neutrophils and memory $\mathrm{T}$ cells to the infected endothelial cells. P-selectin expressed in the stimulated endothelial cells or the activated platelets is stored separately in the Weibel-Palade bodies of endothelial cells and $\alpha$-granules of platelets. P-selectin can move to the cell surface within minutes after stimulation of inflammatory mediators. The function of P-selectin is to mediate endothelial cells adhesion to inflammatory cells and activated platelet adhesion to monocyte and neutrophil. Many studies confirmed that the expression of adhesion molecules increases after renal ischemic/ reperfusion. In a research involving cultured human umbilical vein endothelial cells and human microvascular endothelial cell line in vitro, a lack of oxygen could induce a large number of Weibel-Palade corpuscles in endothelial cells to be released, thereby increasing P-Selectin expression. E-Selectin expression in endothelial cells also increases after hypoxia reoxygenation (27). The kidneys can be protected from ischemic/reperfusion injury by blocking the expression of P-Selectin and E-Selectin $(19,28)$. Klein et al. found that the expression of ICAM-1 does not change significantly during the hypoxic process but increases after hypoxia reoxygenation. Rats with ICAM-1 gene knockout and ICAM-1 monoclonal antibody were used in the studies, which confirmed that ICAM-1 was the key mediator of ischemic AKI and has important role on the kidney I/R injury. Furthermore, 
when ischemia occurred, expression of adhesion molecules increased with the increasing secretion of TNF- $\alpha$ (29) and IL-1 in macrophages and monocytes (30). Increased expression of ICAM-1 induced by IL- $1 \beta$ and TNF- $\alpha$ may lead to an increase of leukocyte infiltration in kidney and synergistically aggravating kidney I/R injury. Zhou (31) used rats to study renal ischemic/reperfusion. He discovered that after ischemia/reperfusion, ICAM-1 expression in renal vascular endothelial cells and P-Selectin expression in renal tubular epithelial cells increase. If anti-P-Selectin antibody was given, kidney I/R injury could be reduced, confirming the important role of ICAM-1 and P-Selectin in kidney I/R injury. The expression of adhesion molecules could be significantly reduced through IP. The results confirmed that preconditioning had a protective effect on reperfusion kidneys. The data in another study confirmed that ischemic preconditioning could prevent postischemic P-selectin expression in the rat small intestine (32). The expression of ICAM-1 of pig lung tissue obviously decreased in group IP than that in control group (33). The study on protective mechanism of ischemic preconditioning in cultured rat endothelial cells found that changes of post-ischemic expression of adhesion molecules such as ICAM-1 may be related to activation of protein kinase $\mathrm{C}$ and production of nitric oxide and free radicals. And this is associated with a lesser adhesion of neutrophils to endothelial cells. Such prevention of neutrophil adhesion may contribute to the protective effect of preconditioning against reperfusion-induced endothelial injury (34). Similar to other methods of blocking the expression of adhesion molecules, we reconfirmed that the increase in adhesion molecule expression plays an important role in kidney I/R injury. Remote ischemic preconditioning can significantly reduce the risk of AKI in patients undergoing cardiopulmonary bypass-assisted cardiac surgery (35). However, the data in some studies indicated that the effect of ischemic preconditioning had limitations and uncertainty. Young et al. reported that 96 adults undergoing high-risk cardiac surgery were randomized to group of remote ischemic preconditioning or control. Main endpoints were plasma high-sensitivity troponin T (hsTNT) levels at 6 and
12 hours, worst post-operative acute kidney injury (AKI). The results showed that there were no significant differences between the experimental and control group, suggesting that IP does not reduce hsTNT, AKI in high-risk cardiac surgery (36).

Compared with other methods that block a certain type of adhesion molecule alone, IP is a much more comprehensive method that can reduce a variety of adhesion molecules with higher precision and reliability. The protective effect of whole kidney can be achieved by ischemia/reperfusion, even the protective effect of other organs can be achieved by controlling inflammatory cytokines. It can also reduce the incidence of multiple organ dysfunction syndrome, which can help meet the needs of clinical and practical use. Unfortunately, some meaningful means of detection can not be performed because of our limited laboratory conditions, making the experimental results in the present study have certain limitations. There also has a certain gap with the complex situation in the actual clinical environment of human body and such findings acquired in the ideal conditions in rats need to further validation at the appropriate internal environment of the human body.

\section{CONFLICT OF INTEREST}

None declared.

\section{REFERENCES}

1. Yin DP, Sankary HN, Chong AS, Ma LL, Shen J, Foster P, et al.: Protective effect of ischemic preconditioning on liver preservation-reperfusion injury in rats. Transplantation. 1998; 66: 152-7.

2. Hausenloy DJ, Yellon DM: Preconditioning and postconditioning: underlying mechanisms and clinical application. Atherosclerosis. 2009; 204: 334-41.

3. Chen X, Liu X, Wan X, Wu Y, Chen Y, Cao C: Ischemic preconditioning attenuates renal ischemia-reperfusion injury by inhibiting activation of IKKbeta and inflammatory response. Am J Nephrol. 2009; 30: 287-94.

4. Murry CE, Jennings RB, Reimer KA: Preconditioning with ischemia: a delay of lethal cell injury in ischemic myocardium. Circulation. 1986; 74: 1124-36. 
5. Przyklenk K, Bauer B, Ovize M, Kloner RA, Whittaker P: Regional ischemic 'preconditioning' protects remote virgin myocardium from subsequent sustained coronary occlusion. Circulation. 1993; 87: 893-9.

6. Hopper RA, Forrest CR, Xu H, Zhong A, He W, Rutka J, et al.: Role and mechanism of PKC in ischemic preconditioning of pig skeletal muscle against infarction. Am J Physiol Regul Integr Comp Physiol. 2000; 279: R666-76.

7. Liu X, Chen H, Zhan B, Xing B, Zhou J, Zhu H, et al.: Attenuation of reperfusion injury by renal ischemic postconditioning: the role of NO. Biochem Biophys Res Commun. 2007; 359: 628-34.

8. Safian RD, Textor SC: Renal-artery stenosis. N Engl J Med. 2001; 344: 431-42.

9. Schrier RW, Wang W: Acute renal failure and sepsis. N Engl J Med. 2004; 351: 159-69.

10. Ojo A0, Wolfe RA, Held PJ, Port FK, Schmouder RL: Delayed graft function: risk factors and implications for renal allograft survival. Transplantation. 1997; 63: 968-74.

11. Perico N, Cattaneo D, Sayegh MH, Remuzzi G: Delayed graft function in kidney transplantation. Lancet. 2004; 364: 1814-27.

12. Ojo AO, Held PJ, Port FK, Wolfe RA, Leichtman AB, Young EW, et al.: Chronic renal failure after transplantation of a nonrenal organ. N Engl J Med. 2003; 349: 931-40.

13. Cochrane J, Williams BT, Banerjee A, Harken AH, Burke TJ, Cairns CB, et al.: Ischemic preconditioning attenuates functional, metabolic, and morphologic injury from ischemic acute renal failure in the rat. Ren Fail. 1999; 21: 135-45.

14. Wever KE, Warlé MC, Wagener FA, van der Hoorn JW, Masereeuw $R$, van der Vliet JA, et al.: Remote ischaemic preconditioning by brief hind limb ischaemia protects against renal ischaemia-reperfusion injury: the role of adenosine. Nephrol Dial Transplant. 2011; 26: 3108-17.

15. Wever KE, Menting TP, Rovers M, van der Vliet JA, Rongen GA, Masereeuw $\mathrm{R}$, et al.: Ischemic preconditioning in the animal kidney, a systematic review and meta-analysis. PLoS One. 2012; 7: e32296.

16. Zhang XL, Selbi W, de la Motte C, Hascall V, Phillips A: Renal proximal tubular epithelial cell transforming growth factor-beta1 generation and monocyte binding. Am J Pathol. 2004; 165: 763-73.

17. Feng L, Ke N, Cheng F, Guo Y, Li S, Li Q, et al.: The protective mechanism of ligustrazine against renal ischemia/ reperfusion injury. J Surg Res. 2011; 166: 298-305.

18. Satoh S, Suzuki A, Asari Y, Sato M, Kojima N, Sato T, et al.: Glomerular endothelium exhibits enhanced expression of costimulatory adhesion molecules, CD80 and CD86, by warm ischemia/reperfusion injury in rats. Lab Invest. 2002; 82: 1209-17.
19. Zhou T, Li X, Wu P, Zhang D, Zhang M, Chen N, et al.: Effect of anti-P-selectin monoclonal antibody on renal ischemia/ reperfusion injury in rats. Chin Med J (Engl). 2000; 113: 790-3.

20. Ogawa T, Nussler AK, Tuzuner E, Neuhaus $P$, Kaminishi M, Mimura $Y$, et al.: Contribution of nitric oxide to the protective effects of ischemic preconditioning in ischemia-reperfused rat kidneys. J Lab Clin Med. 2001; 138: 50-8.

21. Islam CF, Mathie RT, Dinneen MD, Kiely EA, Peters AM, Grace PA: Ischaemia-reperfusion injury in the rat kidney: the effect of preconditioning. Br J Urol. 1997; 79: 842-7.

22. Toosy N, McMorris EL, Grace PA, Mathie RT: Ischaemic preconditioning protects the rat kidney from reperfusion injury. BJU Int. 1999; 84: 489-94.

23. Cochrane J, Williams BT, Banerjee A, Harken AH, Burke TJ, Cairns $\mathrm{CB}$, et al.: Ischemic preconditioning attenuates functional, metabolic, and morphologic injury from ischemic acute renal failure in the rat. Ren Fail. 1999; 21: 135-45.

24. Wever KE, Menting TP, Rovers M, van der Vliet JA, Rongen $\mathrm{GA}$, Masereeuw $\mathrm{R}$, et al.: Ischemic preconditioning in the animal kidney, a systematic review and meta-analysis. PLoS One. 2012;7: e32296.

25. Mahfoudh-Boussaid A, Zaouali MA, Hadj-Ayed K, Miled AH, Saidane-Mosbahi D, Rosello-Catafau J, et al.: Ischemic preconditioning reduces endoplasmic reticulum stress and upregulates hypoxia inducible factor- $1 \alpha$ in ischemic kidney: the role of nitric oxide. J Biomed Sci. 2012; 19: 7.

26. Kim J, Kim JI, Jang HS, Park JW, Park KM: Protective role of cytosolic NADP(+)-dependent isocitrate dehydrogenase, IDH1, in ischemic pre-conditioned kidney in mice. Free Radic Res. 2011; 45: 759-66.

27. Koo DD, Welsh KI, West NE, Channon KM, Penington AJ, Roake JA, et al.: Endothelial cell protection against ischemia/reperfusion injury by lecithinized superoxide dismutase. Kidney Int. 2001; 60: 786-96.

28. Singbartl K, Ley K: Protection from ischemia-reperfusion induced severe acute renal failure by blocking E-selectin. Crit Care Med. 2000; 28: 2507-14.

29. Donnahoo KK, Meng X, Ayala A, Cain MP, Harken AH, Meldrum DR: Early kidney TNF-alpha expression mediates neutrophil infiltration and injury after renal ischemia-reperfusion. Am J Physiol. 1999; 277: R922-9.

30. Savage CO, Brooks CJ, Adu D, Richards G, Howie AJ: Cell adhesion molecule expression within human glomerular and kidney organ culture. J Pathol. 1997; 181: 111-5.

31. Zhou T, Sun GZ, Zhang MJ, Chen JL, Zhang DQ, Hu QS, et al.: Role of adhesion molecules and dendritic cells in rat hepatic/renal ischemia-reperfusion injury and anti-adhesive intervention with anti-P-selectin lectin-EGF domain monoclonal antibody. World J Gastroenterol. 2005; 11: 1005-10. 
32. Davis JM, Gute DC, Jones S, Krsmanovic A, Korthuis RJ: Ischemic preconditioning prevents postischemic P-selectin expression in the rat small intestine. Am J Physiol. 1999; 277: H2476-81.

33. Zhang CF, Chen SX, Guo HZ, Luo WJ: Protective mechanism of ischemic preconditioning to the lung ischemiareperfusion injury. Zhong Nan Da Xue Xue Bao Yi Xue Ban. 2005; 30: 64-7.

34. Beauchamp P, Richard V, Tamion F, Lallemand F, Lebreton $\mathrm{JP}$, Vaudry $\mathrm{H}$, et al.: Protective effects of preconditioning in cultured rat endothelial cells: effects on neutrophil adhesion and expression of ICAM-1 after anoxia and reoxygenation. Circulation. 1999; 100: 541-6.

35. Ferraro PM, Gambaro G: Ischemic preconditioning and the risk of acute kidney injury. Kidney Int. 2012; 82: 243; author reply 243.
36. Young PJ, Dalley P, Garden A, Horrocks C, La Flamme A, Mahon $B$, et al.: A pilot study investigating the effects of remote ischemic preconditioning in high-risk cardiac surgery using a randomised controlled double-blind protocol. Basic Res Cardiol. 2012; 107: 256.

Correspondence address: Dr. Long Liu Department of Urology The General Hospital of Shenyang Military Region Shenyang 110016, China Telephone: +86 24 2885-1201 E-mail: longhecn@163.com 